IRA-International Journal of Applied Sciences ISSN 2455-4499; Vol.04, Issue 03 (2016)

Institute of Research Advances

Pg. no. 431-438

http://research-advances.org/index.php/IRAJAS

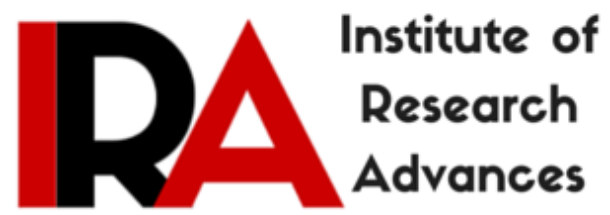

\title{
Promoting Healthy Cognitive Aging: A Systematic Review and Analysis
}

Suk-hee Kim (Ph.D., COI, MSW)

Department of Counseling, Social Work and Leadership

Northern Kentucky University, USA.

Type of Review: Peer Reviewed.

DOI: http://dx.doi.org/10.21013/jas.v4.n3.p7

\section{How to cite this paper:}

Kim, S. (2016). Promoting Healthy Cognitive Aging: A Systematic Review and Analysis. IRA-International Journal of Applied Sciences (ISSN 2455-4499), 4(3), 431438. doi:http://dx.doi.org/10.21013/jas.v4.n3.p7

(C) Institute of Research Advances

\section{(cc) EY-NC}

This work is licensed under a Creative Commons Attribution-Non Commercial 4.0 International License subject to proper citation to the publication source of the work.

Disclaimer: The scholarly papers as reviewed and published by the Institute of Research Advances (IRA) are the views and opinions of their respective authors and are not the views or opinions of the IRA. The IRA disclaims of any harm or loss caused due to the published content to any party. 


\section{ABSTRACT}

Promoting healthy cognitive aging within social work has been raised over the years, but the effectiveness of many preventions and interventions in healthy aging brain has been questioned because of the lack of the evidence. The purpose of the study is to carry out a scientific research review and analysis related to healthy cognitive aging brain and professional social work. This study reviewed major trends in the health care in cognitive aging environment which impacts on social work education, practice, and research including a shift from in-patient to community care setting and increasing diversity of the older adult population. A systematic review was conducted to determine the effectiveness of promoting healthy cognitive aging that target mild-cognitive impairment and screening among olderadults. In this scientific literature review, quantitative and qualitative outcome studies between 1989 and 2016 were reviewed. The researcher found that educational and social activity group interventions that can ease social isolation and loneliness among older adults. However, the effectiveness of home visiting remains unclear. The study also was completed to address a growing area of cognitive aging brain concern and implication for health care providers and professionals. Overall research findings support positive effects of social and family support, early mild-cognitive screening, quality of social network, healthy choice eating, and physical and mental activity in improving healthy mild-cognitive aging.

Key Words: Cognitive Aging, Healthy Cognitive Aging, Older Adults, Mild-Cognitive Function, Social Work

\section{INTRODUCTION}

The lack of cognitive health from mild-cognitive decline to dementia can have profound implication for an individual's health and well-being. As the population ages, the mild-cognitive declinespecifically executive cognitive impairment is an important risk indication that must be assessed in health care settings including medical and community-based mental health fields. Generalist and professional social workers are in a critical position to guide clients about mild-cognitive health promotion and to develop effective preventions and interventions that enhance cognition in the growing aging population.

A literature review was accomplished to provide generalist and professional social workers working in healthy cognitive aging and older adults care with an overview of research related to the promotion of healthy cognitive aging for older adults. The study also was completed to address a growing area of cognitive aging concern and implication for health care providers and professionals. Research analyzing cognitively healthy aging promoting social and family support strategies and the effects on mild-cognitive function of older adults of interventions targeting mild-cognitive screening, social network, healthy eating, and physical, mental and spiritual activity were reviewed. Overall research findings support positive effects of social and family support, early mild-cognitive screening, quality of social network, healthy choice eating, and physical and mental activity in improving healthy mildcognitive aging.

\section{PERCEPTIONS ABOUT COGNITIVE AGING}

\section{Cognitive Aging}

According to Cognitive Aging study in 2015, the term Cognitive Aging is fairly new and less familiar with it then with terms for brain diseases such as dementia and Alzheimer's disease (Blazer, Yaffe, \& Liverman, 2016). We know now much about Alzheimer's disease, but public communication about cognitive aging is becoming interested area in the field of social work. It is much needed to explain how cognitive aging is differs from brain disease, identify the range of cognitive abilities and how they cognitive change with age, and how to maintain cognitive health in aging. A number of studies highlight older Americans' greater fear of dementia and memory loss, compared with other health and financial 
stresses. In 2013 survey supported by the Alzheimer's Association and the Centers for Disease Control and Prevention (CDC) reported that Americans age 60 and older were more afraid of Alzheimer's disease or dementia than of heart disease, stroke, cancer, or diabetes (Alzheimer's Association, 2014).

\section{Mild-Cognitive Impairment}

The U.S. Preventive Services Task Force stated that "Cognitive Impairment is a disorder that causes a person to have problems with memory or other mental activities, like learning, organizing, and making decisions. It includes a range of conditions from mild-cognitive impairment to severe dementia. Alzheimer's disease is one type of cognitive impairment." (2014, pg., 1). Mild cognitive impairment (MCI) is a condition in which people have more memory or other thinking problems than normal for their age, but their symptoms do not interfere with their everyday lives.

Older people with MCT are at greater risk for developing Alzheimer's, but not all of them do. Some studies are underway to learn why some people with MCI progress to Alzheimer's and others do not. The National Institute on Aging study (2016) indicated that "Memory problems can also have other causes, including certain medicines and diseases that affect the blood vessels that supply the brain. Some of the problems brought on by these conditions can be managed or reversed.” (pg., 2). The type of MCI with memory loss as the main symptom is called amnestic MCI. In another type, non-amnestic MCI, the main symptom is an impaired thinking skill other than memory loss, such as trouble planning and organizing or poor judgment.

Some forgetfulness can be a normal part of aging. However, some people have more memory problems than other people their age. This condition is called mild-cognitive impairment, or MCI. People with MCI can take care of themselves and do their normal activities. Here are some examples of MCI memory problems, such as 1) losing things often, 2) forgetting to go to events and appointments, and 3) having more trouble coming up with words than other people of the same age. Memory problems can also have other causes, including certain medicines and diseases that affect the blood vessels that supply the brain. Some of the problems brought on by these conditions can be managed or reversed.

\section{LITERATURE REVIEW ANALYSIS}

\section{Neurocognitive Changes in Aging}

Cognitive change as a normal process of aging has been well documented in the social scientific literature. Some cognitive abilities are strong to brain aging and may even improve with age, such as vocabulary. However, other abilities decline gradually over time. For example, Conceptual reasoning, memory, processing speed (Wisdom, Mignogna, \& Collins, 2012). This study will provide a current brief overview of the neuropsychology of normal cognitive aging.

Cognitive ability can be allocated into specific cognitive domains, such as processing speed, attention, memory, language, visuospatial abilities, and executive functioning or reasoning. Processing speed refers to the speed with which cognitive activities are performed as well as the speed of motor responses. This ability begins to decline in the third decade of life and continues throughout the lifespan (Salthouse, 2010; Salthouse, Fristoe, Lineweaver, \& Coon, 1995; Carlson, Hasher, Zacks, \& Connelly, 1995). The most of cognitive changes reported in healthy older adults are the result of slowed processing speed. The slowing can negatively impact performance on many neuropsychological tests designed to measure other cognitive domains, such as verbal fluency. Therefore, a decline in processing speed can have indications across a variety of cognitive areas.

According to Lezak, Howieson, Bigler, \& Tranel (2012), attention refers to the ability to concentrate and focus on specific stimuli. This ability also shows only a slight decline in late life. 
Selective attention is important for tasks such as engaging in a conversation in a noisy environment or driving a car. This multi-tasking ability is related with working memory. For example, "older adults may have difficulty ordering a numbers in the correct alphanumerical sequence or calculating a tip on a restaurant bill." (Salthouse, Mitchell, Skovronek, \& Babcock, 1989, p.508).

The study by Smith (2016) emphasized that behavioral prevention strategies can help maintain high levels of cognition and functional reliability, and can reduce the social, medical, and economic burden associated with cognitive aging and age-associated diseases. The study also showed interventions involving physical exercise and cognitive training have consistently shown positive effects on cognition in older adults. According to Smith study, Brain fitness interventions have now been shown to have sustained effects lasting 10 years or more (Smith, 2016). Social and behavioral interventions developed and implemented by social workers are important key in supporting and promoting healthy mild-cognitive aging. The study suggested that the National Institutes of Health (NIH) should expand more research on cognitive health and behavioral and social science to promote healthy aging and to develop and cultivate ways to prevent and intervene dementia.

\section{Cognitive Impairment Risk Factors}

The main risk factor for cognitive impairment is getting older. Other risk factors include diabetes, high blood pressure, high cholesterol, tobacco use, alcohol use, depression, and diet and exercise. Mild cognitive impairment involves memory problems that are greater than normal but that do not interfere with a person's ability to carry out usual daily activities. Symptoms of mild-cognitive impairment are difficult to detect because they do not interfere with daily life activities.

The Task Force did find that some screening tools can successfully identify people who have early state dementia. However, the Task Force found no evidence on whether early decision helps clients, caregivers, and doctors make decisions about health care or plan for the future. Therefore, funding for large-scale experimental study is much needed. Also, the Centers for Disease Control and Prevention Healthy Brain Initiative and fund training programs to insure there is a work force with skills to provide high quality care for older adults not only to provide better coverage for behavioral interventions, but also to increase access to evidence-based prevention and healthy aging promotion services with the potential for decreasing dementia high risk.

The Task Force found very little evidence about potential harms of screening for cognitive impairment and dementia. The Task Force also looked at the benefits and harms of drug treatments for cognitive impairment. However, these drugs can have some side effects, including problems sleeping, slow heartbeat, weakness, and upset stomach. The task force stated that some programs for caregivers (for example, family members carrying for people with dementia) can help to reduce burden and depression in a small way.

\section{New Findings of Mild-Cognitive Impairment and Progression to Dementia}

Dr. Roberts and colleagues' examined "2, 719 elderly residents of Olmstead County, MN, every 15 months. The examinations showed that 534 persons had mild-cognitive impairment and some people had MCI when they were first seen where others developed the condition later." (Breitner, J. C. S., 2014, pg., 34). Therefore, their problems were discovered during follow-up exams. The doctors knew from earlier studies that many people with MCI go on to develop dementia after a few years. However, other people show little change over this time, while still other seem to recover to cognitively normal when next examined. (Breitner, 2014).

Why are the findings important? Today, people routinely live into their 70s, 80s, or even 90s. At those ages, problems with memory and other cognitive abilities are so common they are often considered "normal for age." However, many of these people go on to develop dementia. Between beginning 
memory problems and dementia there are in-between conditions that appear to be the first signs of what may become dementia. This means that many people with MCI are actually in transition to dementia. However, other people may have MCI caused by different underlying illnesses. In this case, we want to treat them differently by different underlying illnesses. As Dr. Roberts and colleagues found in their study that all people including those with MCI, have good days and bad days.

\section{Theories of Mild-Cognitive Aging}

The aging is getting older process and degree of age-related changes. Fundamental components of the aging process involve damage from stress, decline in function and cell death. Researchers have suggested that there is selective vulnerability of specific brain regions and systems. For example, the prefrontal cortex, such as leading to impaired executive functioning or subcortical white matter, such as leading to slowed information processing (Gunning et al., 1997).

\section{Early Mild-Cognitive Screening}

Mild Cognitive Impairment (MCI) causes cognitive changes that are serious to be noticed by the individuals experiencing them or to other people. However the changes are not severe enough to interfere with daily life or independent function. Most recent study found that those with MCI have an increased risk of eventually developing Alzheimer's or another type of dementia. It is also important to note that not all people with MCI get worse and some eventually get better. MCI experts classify MCI based on the thinking skills affected. According to the New Diagnostic Criteria and Guidelines for Alzheimer's Disease (2011), The types of MCI are as follow:

1. MCI that primarily affects memory is known as "amnestic MCI." For example, a person may start to forget important information that he or she would previously have recalled easily, such as appointments, conversations or recent events.

2. MCI that affects thinking skills other than memory is known as "nonamnestic MCI." It refers that thinking skills may be affected by nonamnestic MCI include the ability to make sound decisions, judge the time or sequence of steps needed to complete a complex task, or visual perception.

A medical workup for MCI includes the following core elements:

Thorough medical history where the physician documents current symptoms, previous illnesses and medical conditions, and any family history of significant memory problems or dementia.

\section{Social and Family Support Factors Associated with Promoting Healthy Aging}

Some authors used data from more than 800 participants who completed a loneliness scale and found that loneliness was related to the development of incident $\mathrm{AD}$ and to rate of cognitive decline (Maurizio et al., 2016).

\section{Physical, Mental and Spiritual and Healthy Aging}

Currently, the American College of Sports Medicine recommends that exercise programs for older adults include both aerobic and non-aerobic physical activities, such as resistance training, balance training, and stretching for optimal general health. Older adults can benefit a lot from a combination of exercise that can build strength, maintain bone density improve balance, coordination and mobility, reducing the risk of falling, helping maintain independence in performing activities of daily life. One of studies examined the interaction of lifestyle factors and confirmed their positive effects on cognitive functions: physical activity, socialization and reading were associated with better cognitive performance (Maurizio et al., 2016).

\section{Social Network and Healthy Aging}

Some reports have examined the relation of various aspects of social engagement to measures of cognitive and dementia status (Bennett et al., 2014). The finding was robust and persisted after checking 
for numerous activities including social networks, cognitive and physical activities, disability and depressive symptoms.

\section{Healthy Choice Eating and Healthy Aging}

According to the Nutrition and Diet Research (2015), there are all kinds of dietary supplements marketed as brain boosters that promise to preserve cognitive ability as people age. There is plenty evidence that a healthy diet based on whole foods is the best treatment for aging brains. One of the most recent studies, published in the journal Neurology, found that when it comes to eating patterns, a healthy eating style diet could be the most effective choice for reducing individual's risk of cognitive decline as the person get older. The healthier diets among the participants tended to emphasize vegetables, fruits, fish, nuts, soy products, as well as moderate alcohol. The less healthy diets featured the usual suspects, such as red meat, deep-fried foods, and sweets.

\section{IMPLICATION}

The human brain is responsible for all thoughts, feelings, and behaviors. It is command central mission control for the human experience. It is critical since we work with clients to help change their thoughts, feeling, and behavior. It gives us reasons that we should concern ourselves with brain aging and function. However, the social work profession and college and university curricula is lacking in information about the human brain and consequent implications for the assessment, treatment, prevention, and intervention of clients' psychosocial environments.

The lack of cognitive health from mild-cognitive decline to dementia can have profound implication for an individual's health and well-being. As the population ages, the mild-cognitive declinespecifically executive cognitive impairment is an important risk factor that must be assessed in health care settings including community-based mental health fields. Meeting the healthy cognitive aging requires clear and effective communication featuring accurate, up-to-date, and consistent messages that relevant with individuals and their communities. This literature review analysis paid attention to the environmental supports in place to change and maintain behaviors that are supportive of mild-cognitive health. Since new research findings are becoming available, social work generalist and professionals also need reliable means of keeping up with this constantly changing field in gerontology in social work.

\section{RECOMMENDATION}

Overall study suggested that the health care provider can do thinking, memory, and language tests to see if client has MCI. The client may also need to see a specialist for more tests because MCI may be an early sign of Alzheimer's disease. It was strongly recommended that the MCI regular check-up for every 6 to 12 months. The U.S. Preventive Services Task Force has issued a final recommendation statement on Screening for Cognitive Impairment in Older Adults (2014), "adults older than age 65 who do not have recognized signs or symptoms of cognitive impairment. Those who are having problems with memory or thinking should talk to their doctor or nurse. The final recommendation statement summarized what the Task Force learned about the potential benefits and harms of screening for cognitive impairment. However, currently, there is not enough evidence to make a recommendation for or against screening all older adults for cognitive impairment.

Aging is not an issue of just keeping up our own social work profession. It is important to know that it is a professional and ethical mandate to stay current with the research on evidence-based practices and to articulate that information in a way that makes sense to our clients, their families, and health care providers. Promoting healthy cognitive aging and social work model for brain-based social work practice, research and education are strongly recommended in the future study. 


\section{ACKNOWLEDGEMENT}

The author awarded summer fellowship in the summer of 2016 and this study was funded by Northern Kentucky University in Kentucky, USA.

\section{REFERENCES}

Alzheimer's and Dementia. (2014). 2014 Alzheimer's disease facts and figures. The Journal of the Alzheimer's Association, 10(2), 47-92.

Bennett, D. A., Arnold, S.E., Valenzuela, M. J., Brayne, C., \& Schneider, J.A. (2014). Cognitive and social lifestyle: Links with neuropathology and cognition in late life. Acta Neurologica Scandinavica, 127(1), 137-150.

Bauchner, H., Frontanarosa, P.B., \& Golub. R. (2016). The US preventive services task force. The Journal of the American Medical Association, 315(4), 351-352.

Breitner, J. C. S. (2014). Mild cognitive impairment and progression to dementia: New findings. American Academy of Neurology, 82, 34-35.

Blazer, D.G., Yaffe, K., \& Liverman C.T. (Eds.). (2015). Cognitive aging: Progress in understanding and opportunities for action. Washington, DC: National Academies Press.

Carlson, M.C., Hasher, L., Zacks, R. T., \& Connelly, S. L. (1995). Aging, distraction, and the benefits of predictable location. Psychology and Aging, 10, 427-435.

Jack, C. R., Albert, M. S., Knopman, D. S., McKhann, G. M., Sperling, R. A., Carrillo, M. C., Thies, B., $\&$ Phelps (2011). Introduction to the recommendations from the National Institute on Aging. Alzheimer's Association Workgroups on Diagnostic Guidelines for Alzheimer's Disease, 7(3), 257-262.

Lezak, M., Howieson, D., Bigler, E., \& Tranel D. (2012). Neuropsychological assessment. New York: Oxford University Press.

Salthouse, T. (2012). Consequences of age-related cognitive declines. Annual review of psychology, 63, 201-226.

Salthouse, T. A. (2010). Selective review of cognitive aging. Journal of the International Neuropsychological Society, 16, 754-760.

Salthouse, T. A., Mitchell, D. R., Skovronek, E., \& Babcock, RL (1989). Effects of adult age and working memory on reasoning and spatial abilities. Journal of Experimental Psychology Learning, Memory, and Cognition, 15, 507-516.

Smith, G. E. (2016). Healthy cognitive aging and dementia prevention. American Psychologist, 7(4), 268275.

Raz, N., Gunning, F. M., Head, D., Dupuis, J. H., McQuain, J., Briggs, S. D., Loken, W. J., Thornton, A.E., \& Acker, J.D. (1997). Selective aging of the human cerebral cortex observed in vivo: Differential vulnerability of the prefrontal gray matter. Cereb Cortex, 7, 268-282. 
The National Institute on Aging's ADEAR Center. (2016). Forgetfulness: Knowing when to ask for help. The National Institute on Aging, 2-12.

The U.S. Preventive Services Task Force. (2014). Screening for cognitive impairment in older adults. Understanding Task Force Recommendations, 1-3.

Williams, K. N. \& Kemper, S. (2010). Interventions to reduce cognitive decline in aging. Journal of Psychosocial Nursing and Mental Health Services, 48(5), 42-51.

Wisdom, N. M., Mignogna, J., \& Collins, R. L. (2012). Variability in Wechesler Adult Intelligence ScaleIV subtest performance across age. Archives of clinical neuropsychology: The Official Journal of the National Academy of Neuropsycholigists, 27, 389-397. 